\title{
Energy storage day-ahead scheduling to reduce grid energy export and increase self-consumption for micro-grid and small power park applications.
}

\author{
Joanna Sobon \\ University of Strathclyde, UK \\ E-mail \\ joanna.sobon@strath.ac.uk
}

\author{
Andrew Roscoe \\ University of Strathclyde, UK \\ E-mail \\ andrew.j.roscoe@strath.ac.uk
}

\author{
Bruce Stephen \\ University of Strathclyde, UK \\ E-mail \\ bruce.stephen@strath.ac.uk
}

\begin{abstract}
Developments in energy storage technology will start to play a prominent role in overcoming the problems of generation intermittency by providing the ability to shift demand to times when generation is available. However, exploiting the potential of this technology requires the design of an optimal charging and discharging schedule to allow its integration with the energy network that brings maximum advantage to both the system and the user. This paper introduces a mathematical model for generation and demand forecasting with energy storage scheduling that can be used for micro-grid and small power park applications. The proposed solution models the physical limitations associated with the energy storage technology used, which will constrain charge and discharge schedules beyond what can be forecast for them. A case study of a community feeder with large PV installations is presented to demonstrate the effectiveness of the model. Day-ahead charge and discharge schedules were produced that increased self-consumption within the system and reduced energy export to the grid. The main contribution of this work is the design of a generic parametrized forecasting and energy storage scheduling tool that will be a platform for further development to specialized storage technology and its potential scalability.
\end{abstract}

Index Terms-- Energy storage, discharge schedule, microgrid, demand forecasting, generation forecasting, Power Park.

\section{NOMENCLATURE}

$E_{\text {stored }}(t)$ Energy stored in the electrical energy storage at time $\mathrm{t}[\mathrm{kWh}]$

SOC State of charge of the electrical energy storage $[\mathrm{kWh}]$

$E_{\text {storage flow }}$ Amount of energy that is exchanged with energy storage and rest of the system [kWh]

$E_{\text {flow_max }} \quad$ Maximum energy that can flow to the storage [kWh]

$E_{\text {flow_min }} \quad$ Minimum energy that can flow to the storage [kWh]

$P_{\text {rated }} \quad$ Rated power of the energy storage $[\mathrm{kW}]$

$t \quad$ Time index

$\Delta t \quad$ Time interval

$\alpha \quad$ Self-discharge rate of the energy storage

$\eta \quad$ Electrical energy storage efficiency

$M A P E \quad$ Mean absolute percentage error
$G P$

$G B M$

$N N$

ARIMA

EES

\author{
Gaussian Process \\ Gradient boost machine \\ Neural Network \\ Autoregressive Integrated Moving Average \\ Electrical energy storage
}

\section{INTRODUCTION}

Currently, the GB power network is facing many challenges due to undergoing changes dictated by environmental protection issues, depleting non-renewable energy resources, the necessity of improving the security of the supply. The shifting from conventional power network architecture, where power generation is centralised and located far from the enduser, to the smart grid, characterised by generation distributed within the network and a great share of renewable energy generation, sets many challenges that need to be resolved to ensure reliability and stability of the system to be maintained. An increasing penetration of the renewable energy generation in power network introduces uncertainty about energy available to maintain a balance between generation and demand [1]. This type of generation provides time fluctuating output strongly dependent on the weather. Accurate and timely forecasting of demand and generation with resulting demand side response actions could enhance the reliability of small power systems with high penetrations of embedded renewable generation [2]. With the development in energy storage technology, this device has potential to become a key element of new power network as described in the UK government's National Infrastructure Commission Smart Power Report [3]. The storage can not only play prominent role in overcoming the problems of generation intermittency by providing the ability to shift demand to times when generation is available, but can also provide the ability to shift the power from off-peak hours to meet demand during peak hours by storing excess of energy and releasing it when required [4-6]. The other support services that energy storage can provide are frequency response services, reserve capacity, regulation and other [7]. However, exploiting the potential of this technology requires the design of an optimal charging and discharging schedule to allow its integration with the energy network that brings maximum advantage to both the system and the user. Optimal operation of the energy storage is a very complex problem. Many factors need to be 
considered to ensure that this device works in a way that optimally uses its advantages and gives an economically feasible solution. These are the life span of the energy storage and its technical limitations, the connection location of storage in the power network [5, 8, 9], the main purpose of this device in this configuration $[5,6]$. Research in electrical energy storage area is focused on the optimal sizing $[9,10]$, operation [11], scheduling [12] and control [13] to allow its successful integration with the power network.

The main contribution of the work presented in this paper is the design of generic parametrized forecasting and energy storage scheduling tool for small power parks and micro-grid applications. This produces day-ahead energy storage charging and discharging schedule that decreases electrical energy export to the main grid and increases selfconsumption of energy resources within the system. The proposed model constitutes a platform for further development to specialized storage technology and its potential scalability.

The remaining part of the paper is structured in following way. Section III describes model formulation and implementation. The case study and evaluation of the performance and the effectiveness of proposed model is performed in section IV. Finally, the last section concludes a paper and includes recommendations for further model improvements.

\section{MODEL FORMULATION AND IMPLEMENTATION}

A grid-connected system consisting of the electrical load, PV generation, and electrical energy storage is modelled which includes the energy storage scheduler that controls energy flow within the system to achieve a reduction in export to the grid and increase in self-consumption. The energy flow in the developed model is presented in Fig. 1.

\section{A. Forecasting of demand and generation models}

Accurate and timely forecasting of demand and forecasting of renewable generation has an important influence on the operation of the power network. Due to change in the infrastructure of the power network, the short time demand forecasting becomes more challenging. Since this is moving closer to end-use and the load on local and disaggregated levels has more volatile and noisy characteristics, it is more difficult to predict [14]. With the decrease in load aggregation, the forecasting error increases significantly from around $3 \%$ for National level, through $10 \%$ for the secondary substation to $30 \%$ for end-user $[14,15]$. The developed model
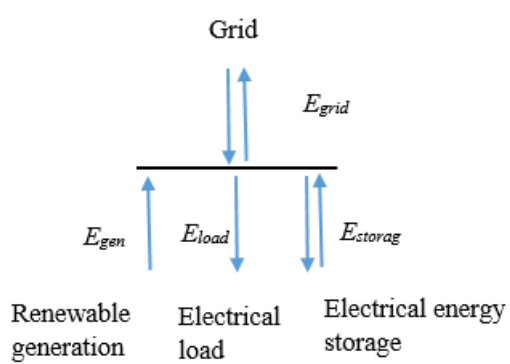

Fig. 1. Energy flow in the modelled system. makes use of short time demand forecasting method previously used in this area of research. The different methods of demand forecasting are employed to enable investigating the influence of used forecasting models on the performance of developed EES schedule tool. Due to lack of the weather data, the purely data-driven forecasting methods are used.

1) Demand forecasting: Different methods of demand forecasting are implemented in the model. These are an ARIMA model with lags of 48 samples, and feed-forward Neural Network with 48 output nodes and 49 input nodes as used in [16], model based on the Gaussian Process (GP) and Gradient Boost Machine (GBM) used by [17], Ensemble Forecast (combination of all used demand forecasting methods by simple unweighted average) [18, 19] and finally Persistent forecasting.

2) PV generation forecasting: The PV generation forecasting, used in this work, is based on the persistence model. This naïve method assumes that the energy generated today at given time is the same as it was yesterday at the corresponding time.

\section{B. Electrical Energy Storage Model}

The parametrized model of electrical energy storage based on the energy balance equation (1) was developed.

$$
\mathrm{E}_{\text {stored }}(\mathrm{t}+1)=(1-\alpha) * \mathrm{E}_{\text {stored }}(\mathrm{t})-\eta^{*} \mathrm{E}_{\text {storage_flow }}(\mathrm{t})
$$

Where the energy storage reduces the input energy to the system by the value associated with self-discharge rate for given interval of time and the efficiency associated with the charging and discharging processes of energy storage reduces the potential energy that can flow from and to the storage. During model development, the following parameters were considered: state of charge of EES, minimum and maximum state of charge, rated power, capacity, efficiency and self-discharge rate. The energy flow from storage at any given interval of time in proposed model is constrained by rated power of used storage technology as follows in (2).

$$
\mathrm{E}_{\text {flow_min }} \leq \mathrm{E}_{\text {storage_flow }}(\mathrm{t}) \leq \mathrm{E}_{\text {flow_max }}
$$

Where:

$$
\begin{aligned}
& \mathrm{E}_{\text {flow_max }}=\mathrm{P}_{\text {rated }} * \Delta \mathrm{t} \\
& \mathrm{E}_{\text {flow_min }}=-\mathrm{P}_{\text {rated }} * \Delta \mathrm{t}
\end{aligned}
$$

Additionally, the state of charge of energy storage is limited by maximum and minimum allowed values (5) resulting from storage specification.

$$
\mathrm{SOC}_{\min } \leq \mathrm{SOC}(\mathrm{t}) \leq \mathrm{SOC}_{\max }
$$

Where the $\mathrm{SOC}_{\max }$ is related to the maximum allowed level of charge of the EES and $\mathrm{SOC}_{\min }$ is the minimum allowed the depth of discharge for simulated technology.

\section{Scheduling of Energy Storage System}

The day-ahead scheduling of the electrical energy storage is performed basing on the forecasted daily profile of facility electricity demand and renewable generation forecast. The output of the scheduling are periods of time and corresponding actions to be 
performed by EES during these periods (charge, discharge or "no action"). The logic that controls charge and discharge regimes is based on the available renewable energy resources in the system and technical limitation of the energy storage to be simulated. The main aim of the algorithm controlling the schedule is to reduce export energy to the grid and increase self-consumption within the system.

\section{CASE STUDY AND RESUlTS}

To assess performance and effectiveness of the proposed model the case study of an actual community feeder with significant PV installation is presented. Half-hourly historical data of electrical demand and PV generation covering 126 days were used. The weather data for this period and location were not available. Dataset was divided into two subsets to allow implementation and testing of the demand forecasting method used in this work.

\section{A. Effect of demand forecasting methods accuracy on the energy storage model performance}

Six simulations, with different forecasting methods employed, were considered to study the effect of the error in demand forecasting on the level of reduction in electricity export to the grid and the increase of self-consumption within the simulated system. A 40kWh lead acid battery ESS with rated power of $10 \mathrm{~kW}$, efficiency $75 \%$ and discharge rate of 0.005 per half-hour was considered. The results of simulations are given in Table I. The example of the electrical energy storage charging and discharging schedule produced by the model is illustrated in Fig. 2. The reduction in grid export achieved by developed model is in the range of $71.4 \%$ $-82.3 \%$ and the self-consumption is increased by between 70 $78.3 \%$ with the model employing persistence and GP demand forecasting model archiving the best results for the export reduction and self-consumption increase respectively. The worst performance in both cases achieves the model employing $\mathrm{NN}$ demand forecast. The simulation results show that there is a strong negative correlation between the forecasting error and the reduction in export to the grid and moderate negative correlation with the increase in selfconsumption, equal -0.93 and -0.67 respectively. It indicates that accuracy of employed forecasting method has a significant influence on model performance. The naïve method, Persistence demand forecasting used in the developed tool, gives the higher export reduction among all

TABLE I

PERFORMANCE OF SCHEDULER MODEL

\begin{tabular}{|c|c|c|c|}
\hline \multirow[t]{3}{*}{ Forecasting model } & \multirow{2}{*}{ 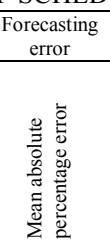 } & \multicolumn{2}{|c|}{$\begin{array}{c}\begin{array}{c}\text { Performance of scheduler } \\
\text { model }\end{array} \\
\end{array}$} \\
\hline & & 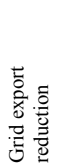 & 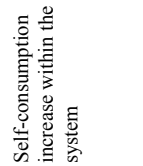 \\
\hline & [\%] & {$[\%]$} & {$[\%]$} \\
\hline Ensemble forecast (all) & 27.4 & 80 & 75.6 \\
\hline Gradient boost machine & 29 & 79.1 & 78.3 \\
\hline Persistence forecast & 29.7 & 82.3 & 73.2 \\
\hline Gaussian process & 30.6 & 80.3 & 76.5 \\
\hline ARIMA model & $\frac{3.0}{48.2}$ & 74.9 & 74.6 \\
\hline Neural networks & 50 & 71.4 & 70 \\
\hline
\end{tabular}

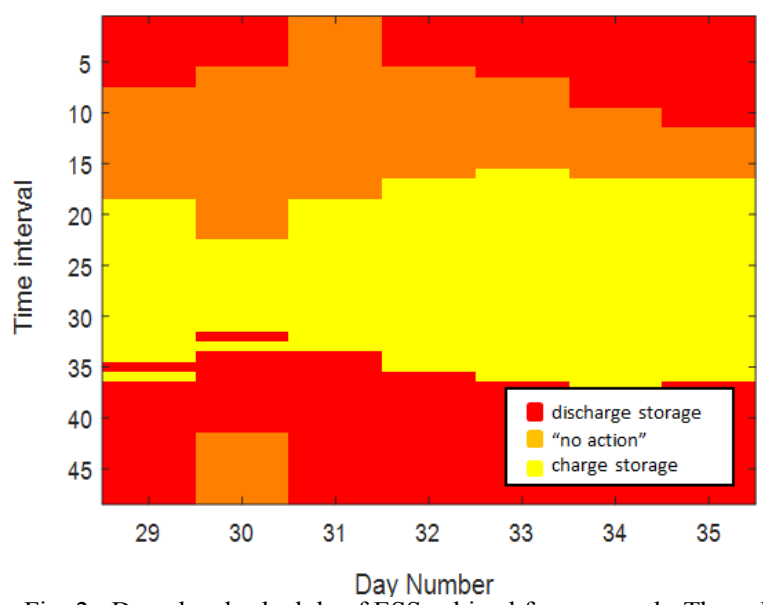

Fig. 2. Day-ahead schedule of ESS achived for one week. The colors indicates the appropriate action to be performed by ESS. Colors represent charging, discharging and "no action" function of energy storage, yellow, red and orange respectively.

used models, but it does not perform so well with the increase of self-consumption. The model employing Ensemble forecast has the lowest mean absolute percentage error but does not introduce the higher percentage increase in the analysed quantities than other models. It indicates that other performances of the forecasting method need to be analysed and their influence on the performance of the developed model should be evaluated.

\section{B. Evaluation of the energy storage scheduler effectiveness}

The analysis of the energy storage scheduler effectiveness in predicting required storage actions is presented in this section. The method based on the confusion matrix is proposed. The confusion matrix is the table of true and false positives (TP, FP respectively) and true and false negatives (TN, FN respectively) that are results of comparing the outcomes of classification, hypothesis testing, object/event detection with the available target/ true/ actual outcomes [20]. It is a way to describe the performance of the classification model or classifier [20,21]. The example of confusion matrix with its notation is shown in Fig. 3. The rows of the matrix represent the actual (target) occurrences of the particular storage action, and the columns correspond to predicted instances of this action. This method enables to evaluate the scheduler model capability to predict the occurrence of a particular action of the electrical energy storage. In this work, the target value was schedule

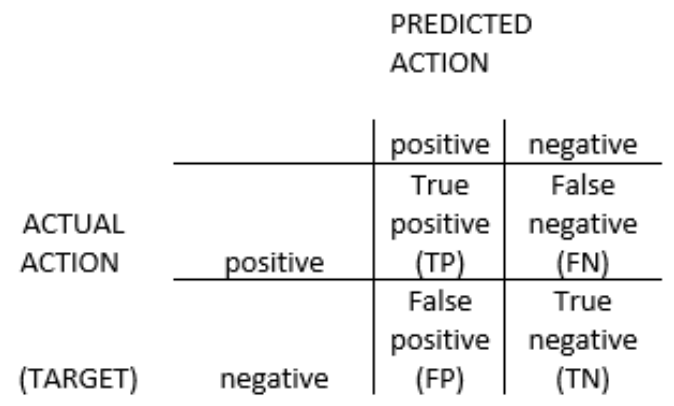

Fig. 3. Confusion matrix notation. 
when actual demand data were used during simulation. The predicted results came from simulations with different demand forecasting methods employed in the model. For each case, the three separate matrices were prepared for predicting charging, discharging and the "no action" state with a comparison to the target schedule. The example of confusion matrices for a schedule when the GP demand forecasting was employed within the model is shown in Fig. 4. The assessment of the effectiveness of the schedule was performed basing on different performance measures derived from those matrices such as sensitivity, specificity, false positive rate, precision/positive predicted value. All these indicators 'describe the level at which the evaluated classifier succeeds a fail to correctly detect a positive class' [21]. The sensitivity and the specificity give the proportion of actual instances, which are correctly identified, positive in case of sensitivity and negative for specificity. False positive rate gives knowledge about the number of negative occurrences that was predicted to be positive. Finally, precision is the proportion of positive instances that were correctly identified. $[20,21]$. Base on the confusion matrix notation from Fig. 3. all mentioned above indices can be computed as follows.

$$
\begin{aligned}
& \text { sensitivity }=\mathrm{TP} /(\mathrm{TP}+\mathrm{FN}) \\
& \text { specificity }=\mathrm{TN} /(\mathrm{TN}+\mathrm{FP}) \\
& \text { false positive rate }=\mathrm{FP} /(\mathrm{TN}+\mathrm{FP}) \\
& \text { precision }=\mathrm{TP} /(\mathrm{TP}+\mathrm{FP})
\end{aligned}
$$

As stated in [21] using all performance measures derived from the confusion matrix in the same time leads to significant informational redundancy. Usually, the performance measure is performed by assessing a complementary pair of indicators such as sensitivity with specificity, precision with sensitivity and true positive rate with the false positive rate. It is because one of the indicators describes the ability to detect the positive occurrence and other one describes capability to detect negative instances [21]. In

\begin{tabular}{|c|c|c|c|}
\hline & \multicolumn{3}{|c|}{$\begin{array}{l}\text { PREDICTED } \\
\text { ACTION }\end{array}$} \\
\hline & discharge & yes & no \\
\hline ACTUAL & yes & 922 & 124 \\
\hline \multirow[t]{3}{*}{ ACTION } & no & 109 & 1534 \\
\hline & & \multicolumn{2}{|c|}{$\begin{array}{l}\text { PREDICTED } \\
\text { ACTION }\end{array}$} \\
\hline & charge & yes & no \\
\hline ACTUAL & yes & 851 & 49 \\
\hline \multirow[t]{3}{*}{ ACTION } & no & 66 & 1723 \\
\hline & & \multicolumn{2}{|c|}{$\begin{array}{l}\text { PREDICTED } \\
\text { ACTION }\end{array}$} \\
\hline & "no action" & yes & no \\
\hline ACTUAL & yes & 644 & 99 \\
\hline ACTION & no & 97 & 1849 \\
\hline
\end{tabular}
this work, the analysis of sensitivity and specificity is carried out to evaluate the schedule effectiveness.

When selecting the best classification method, ideally, both

Fig. 4. The confusion matrices for charging, discharging and "no action" state prediction of energy storage schedule for model employing the Gaussian Process demand forecasting. its sensitivity and specificity should be high; in practice, it is a compromise based on application specific criteria. The method with very high sensitivity does not perform well if it is characterised by poor specificity and vice versa. These two quantities have complementary character thus it is necessary to achieve high values for both to ensure effectiveness in classification. The sensitivity indicates how good a method is in predicting positive outcome and the specificity gives information on how good the model performs with the prediction of "not performing this action." The higher value of this second indicator, the fewer cases that the action is predicted to be performed even if it should not be. For the EES it is crucial that the charging and the discharging action are not performed when they should not be because it puts the EES in a position that it becomes not capable to achieve system goals of reducing export to the grid and increasing the self-consumption within the system. For this reason, the specificity is treated as the leading criterion for assessing the schedule effectiveness in this work and the sensitivity is treated as the subsequent criterion.

In the presented work, the effectiveness of the scheduler model employing a different method of demand forecasting was evaluated. The analysis of effectiveness was based on the performance of prediction for the three possible actions that storage can perform: discharge, charge or do not perform an action in given interval of time. The calculated performance measures for all possible simulated cases were summarised in Table II. According to II, the scheduler model employing ensemble forecast and GP forecast of demand achieve the highest specificity in the prediction of the energy storage actions except predicting "no action" state for which the model employing GBP demand forecasting performs the best. The scheduler model that uses Gaussian Process regression to forecast demand was chosen as the potential candidate with the good effectiveness in the charge and the discharge action prediction. It also achieves the higher sensitivity in predicting "no action" state than remaining two above mentioned models. Additionally, analysis of the model performance in the grid export reduction and self-consumption increase for the model employing GP, GBM and ensemble forecast, can

\begin{tabular}{|c|c|c|c|c|c|c|c|c|}
\hline \multirow[t]{2}{*}{ Action } & \multirow{2}{*}{\multicolumn{2}{|c|}{ Described parameter }} & \multicolumn{6}{|c|}{ Demand forecasting model used } \\
\hline & & & 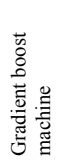 & 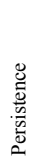 & 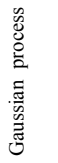 & 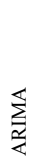 & 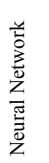 & 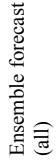 \\
\hline \multirow{4}{*}{ 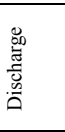 } & Sensitivity & \multirow{4}{*}{ (\%) } & 89.1 & 87.3 & 88.1 & 77.3 & 70.9 & 88.4 \\
\hline & Specificity & & 92.9 & 91.9 & 93.4 & 87.8 & 92.1 & 93.4 \\
\hline & $\begin{array}{l}\text { False positive } \\
\text { rate }\end{array}$ & & 7.1 & 8.1 & 6.6 & 12.2 & 7.9 & 6.6 \\
\hline & Precision & & 88.8 & 87.3 & 89.4 & 80.2 & 85.2 & 89.5 \\
\hline \multirow{4}{*}{ 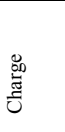 } & Sensitivity & \multirow{4}{*}{ (\%) } & 294.9 & 93.1 & 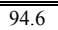 & 89.2 & 84.9 & 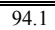 \\
\hline & Specificity & & 96 & 96.2 & 96.3 & 92.1 & 94.1 & 96.3 \\
\hline & $\begin{array}{l}\text { False positive } \\
\text { rate }\end{array}$ & & 4 & 3.8 & 3.7 & 7.9 & 5.9 & 3.7 \\
\hline & Precision & & 92.3 & 92.5 & 92.8 & 85 & 87.8 & 92.7 \\
\hline \multirow{4}{*}{$\begin{array}{l}\tilde{0} \\
\stackrel{0}{0} \\
0 \\
\check{z}\end{array}$} & $\begin{array}{l}\text { Sensitivity } \\
\end{array}$ & \multirow{4}{*}{ (\%) } & 84.9 & 84.4 & 86.7 & 74.4 & 90.6 & 86.4 \\
\hline & Specificity & & 95.7 & 94.3 & 95 & 90.6 & 85.9 & 94.9 \\
\hline & $\begin{array}{l}\text { False positive } \\
\text { rate }\end{array}$ & & 4.3 & 5.7 & 13.35 & 9.4 & 14.1 & 5.1 \\
\hline & Precision & & 88.3 & 85.1 & 27.6 & 75.2 & 71 & 86.5 \\
\hline
\end{tabular}

TABLE II

PERFORMANCE MEASURE VALUES FOR PREDICTION OF ACTION OF THE ENERGY STORAGE DEVICE 
support this decision. The model using GP forecast achieves the highest export reduction and the second best result in selfconsumption increase comparing the performance of these three models. The next reason for choosing this method as the most promising is that it requires less computation during proceeding forecast than ensemble forecast.

Not necessarily the most accurate forecasting of demand gives the most effective schedule for all possible EES actions. It indicates that further work on the influencing factors on the schedule effectiveness should be performed. Furthermore, the influence of performance of forecasting model used in the scheduling tool should be more closely investigated.

\section{Effect of electrical energy storage size on developed model performance}

The effect of the size of energy storage on the increase in the self-consumption and reduction of the export was investigated. The ensemble demand forecasting was employed in the model for this set of simulations. Energy storage technology with parameter described in section A except for the capacity which was varied between $10 \mathrm{kWh}$ and $200 \mathrm{kWh}$ was used during simulations. Fig. 5. illustrates an increase in the size of storage, up to $50 \mathrm{kWh}$, resulting in significant increase in self-consumption and significantly reduced energy export to the grid. Self-consumption increased by $62.6 \%$ when the capacity of the electrical energy storage was gradually enlarged from $10 \mathrm{kWh}$ to $50 \mathrm{kWh}$. Simultaneously, the grid export reduction was increased by $66.3 \%$. When the capacity of the storage was further increased, the rate of change in self-consumption increase and the export reduction significantly decreased and achieved $8 \%$ for both quantities. There were not significant changes in storage performances observed for storage with capacity above $80 \mathrm{kWh}$. The self-consumption reached $94.3 \%$ increase and grid export reduction achieved $100 \%$ for capacity above

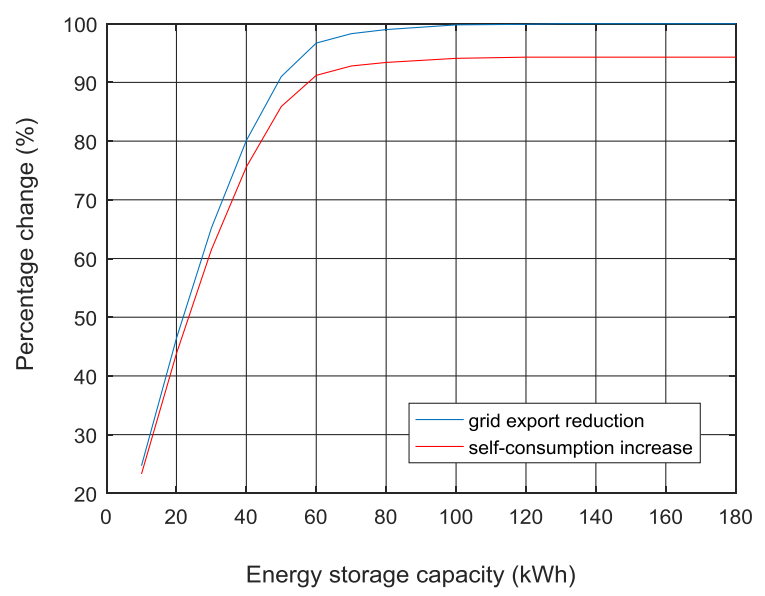

Fig. 5. Grid export reduction and the increase in self-consumption versus energy storage capacity. The blue line indicates the change in the energy export to the main grid and the red one presents the selfconsumption increase.
$80 \mathrm{kWh}$. Since electrical energy storage technology is still very expensive, it is crucial from the economical point of view to install storage with capacity, which will be fully employed during operation. Additionally, installation of such technology needs to bring maximum technical benefits. It indicates the necessity of optimal sizing of the EES that bring maximum advantage to the system and gives an economically feasible solution.

\section{CONCLUSION AND RECOMMENDATION FOR MODEL IMPROVEMENTS}

This paper has presented an algorithm for producing oneday ahead electrical energy storage charging and discharging schedules that reduce the energy export to the grid and additionally increase self-consumption in the modelled system. Depending on the forecasting of demand method employed in the model the energy export to the grid was reduced by between $71.4 \%-82.3 \%$, and self-consumption was increased by $70 \%-78.3 \%$. The strong correlation between mean absolute percentage error of the forecasting method used and export reduction indicates that a forecasting method with low MAPE is preferred as it can improve the overall storage system performance.

The evaluation of the effectiveness of EES schedule leads to the conclusion that the most promising model from all considered was those employing Gaussian processes to forecast demand. This model was the most effective in predicting of charge and discharge actions of energy storage and second best in predicting "no action" state. Additionally, it achieved higher export to the grid reduction and greater increases in self-consumption than equivalent model employing ensemble forecast.

The analysis of the size of the EES on potential reduction in grid export shows that increasing the size of the storage leads to the increase in reduction of export to the grid up to the point when the available storage capacity will not be fully employed during operation due to lack of renewable generation. Installing the storage with too high capacity can significantly increase the cost leading to economically infeasible solutions indicating the necessity to introduce optimal sizing of the ESS within further model developments.

\section{REFERENCES}

D. A. Halamay, T. K. A. Brekken, A. Simmons, and S. McArthur, "Reserve Requirement Impacts of Large-Scale Integration of Wind, Solar, and Ocean Wave Power Generation," IEEE Transactions on Sustainable Energy, vol. 2, no. 3, pp. 321-328, 2011.

A. J. Roscoe and G. Ault, "Supporting high penetrations of renewable generation via implementation of real-time electricity pricing and demand response," IET Renewable Power Generation, vol. 4, no. 4, pp. 369-382, 2010.

"Smart Power," A National Infrastructure Commission Report, 2016, [Online], Available: https://www.gov.uk/government/publications/smart-power-anational-infrastructure-commission-report.

[4] J. P. Barton and D. G. Infield, "Energy storage and its use with intermittent renewable energy," Energy Conversion, IEEE Transactions on, vol. 19, no. 2, pp. 441-448, 2004. 

Proto, "Optimal Integration of Distributed Energy Storage Devices in Smart Grids," IEEE Transactions on Smart Grid, vol. 4, no. 2, pp. 985-995, 2013

[6] D. Greenwood, N. Wade, P. Taylor, P. Papadopoulos, N. Heyward, and S. Alilat, "A Forecasting, Optimization and Scheduling System for Energy Storage Systems in distribution networks," in 2016 IEEE Power and Energy Society General Meeting, PESGM 2016, July 17, 2016 - July 21, 2016, Boston, MA, United states, 2016, vol. 2016-November: IEEE Computer Society.

[7] G. H. Abbas A. Akhil, Aileen B. Currier, Benjamin C. Kaun, Dan M. Rastler, and A. L. C. Stella Bingqing Chen, Dale T. Bradshaw, and William D. Gauntlett, "DOE/EPRI 2013 Electricity Storage Handbook in Collaboration with NRECA," United States Department of Energy by Sandia CorporationJuly 2013

[8] M. C. Bozchalui and R. Sharma, "Optimal operation of Energy Storage in distribution systems with Renewable Energy Resources," in 2014 Clemson University Power Systems Conference, 2014, pp. 1-6.

[9] S. B. Karanki and D. Xu, "Optimal capacity and placement of battery energy storage systems for integrating renewable energy sources in distribution system," in 2016 National Power Systems Conference (NPSC), 2016, pp. 1-6.

[10] J. P. Fossati, A. Galarza, A. Martín-Villate, and L. Fontán, "A method for optimal sizing energy storage systems for microgrids," Renewable Energy, vol. 77, pp. 539-549, 5// 2015.

[11] G. Carpinelli, S. Khormali, F. Mottola, and D. Proto, "Optimal operation of electrical energy storage systems for industria applications," in 2013 IEEE Power \& Energy Society General Meeting, 2013, pp. 1-5

[12] H. Farzin, M. Fotuhi-Firuzabad, and M. Moeini-Aghtaie, "A stochastic multi-objective framework for optimal scheduling of energy storage systems in microgrids," IEEE Transactions on Smart Grid, vol. PP, no. 99, 2016.
M. Rowe, W. Holderbaum, and B. Potter, "Control methodologies: Peak reduction algorithms for DNO owned storage devices on the Low Voltage network," in IEEE PES ISGT Europe 2013, 2013, pp. 1-5.

B. Hayes, J. Gruber, and M. Prodanovic, "Short-Term Load Forecasting at the local level using smart meter data," in 2015 IEEE Eindhoven PowerTech, 2015, pp. 1-6.

M. Ghofrani, M. Hassanzadeh, M. Etezadi-Amoli, and M. S. Fadali, "Smart meter based short-term load forecasting for residential customers," in 2011 North American Power Symposium, 2011, pp. 1-5.

A. Marinescu, C. Harris, I. Dusparic, S. Clarke, and V. Cahill, "Residential electrical demand forecasting in very small scale: An evaluation of forecasting methods," in 2013 2nd International Workshop on Software Engineering Challenges for the Smart Grid (SE4SG), 2013, pp. 25-32.

J. R. Lloyd, "GEFCom2012 hierarchical load forecasting: Gradient boosting machines and Gaussian processes," International Journal of Forecasting, vol. 30, no. 2, pp. 369-374, 2014.

C. E. Borges, Y. K. Penya, and I. Fernandez, "Evaluating Combined Load Forecasting in Large Power Systems and Smart Grids," Industrial Informatics, IEEE Transactions on, vol. 9, no. 3, pp. 1570-1577, 2013.

B. Stephen, X. Tang, P. R. Harvey, S. Galloway, and K. I. Jennett, "Incorporating practice theory in sub- profile models for short term aggregated residential load forecasting," IEEE Transactions on Smart Grid, vol. PP, no. 99, 2015, pp.1-8.

K. P. Murphy, Machine learning : a probabilistic perspective. Cambridge, Mass.: Cambridge, Mass. : MIT Press, 2012.

P. Cichosz, Data mining algorithms : explained using $R /$ [internet resource]. Chichester, West Sussex ; Malden, MA : John Wiley \&amp; Sons Inc., 2015. 